\title{
Factores claves del éxito de las organizaciones que han adoptado la norma ISO 9001
}

\section{Key factors in the success of organizations that have adopted ISO 9001}

\begin{abstract}
Alexis Fernando Bolaños Jijón
Universidad de Guayaquil, Ecuador

Mónica Marlene Baquerizo Anastacio

Universidad de Guayaquil, Ecuador

Autor para correspondencia: alexis.bolanosj@ug.edu.ec, monica.baquerizoa@ug.edu.ec Fecha de recepción: 28 de Agosto 2017 - Fecha de aceptación: 15 de Enero de 2018
\end{abstract}

Resumen: La Calidad es fundamental para que las organizaciones puedan obtener resultados. En la historia, la calidad ha evolucionado de tal manera que en la actualidad se han desarrollado algunas filosofías, entre ellas la Gestión Total de la Calidad (TQM), para permitir a las organizaciones cumplir con su propósito. Sin embargo, como toda filosofía, TQM requiere de modelos y lineamientos que permitan ser asumidos por las organizaciones y obtengan los beneficios que esperan. Uno de esos modelos es la norma ISO 9001, la más reconocida a nivel mundial, que promueve beneficios a través del cumplimiento de los requisitos de esta norma y la mejora continua del sistema de gestión de calidad. A pesar de que existen abundantes estudios acerca de los factores clave del éxito de TQM en las organizaciones, no se considera conveniente asumirlos para la norma ISO 9001, debido que ésta tiene una estructura y propósitos particulares que requieren ser tomados en cuenta. A través de la revisión de literatura pertinente, en este artículo se propusieron factores claves para alcanzar el éxito de los sistemas de gestión de calidad de las organizaciones basados en la norma ISO 9001.

Palabras claves: calidad; gestión total de la calidad; sistema de gestión de calidad; iso 9001; factores clave

\begin{abstract}
Quality is fundamental for organizations to achieve results. In history, quality has evolved in such a way that some philosophies have been developed, including Total Quality Management (TQM), to enable organizations to fulfill their purpose. However, like all philosophy, TQM requires models and guidelines that allow the organizations to assume the benefits they expect. One such model is ISO 9001, the most recognized worldwide, which promotes benefits through compliance with the requirements of this standard and the continuous improvement of the quality management system. Although there are many studies on the key factors of TQM's success in organizations, it is not considered appropriate to assume them for ISO 9001, since it has a particular structure and purposes that need to be taken into account. Through the review of pertinent literature, this article proposed key factors to achieve the success of the quality management systems of organizations based on ISO 9001.
\end{abstract}

Key Words: quality; total quality management; quality management system; iso 9001; key factors 


\section{Introducción}

La Calidad es fundamental para que las organizaciones puedan obtener resultados tanto en rentabilidad como en aumento de la participación en el mercado (Evans \& Lindsay, 2014) y por este motivo las organizaciones excelentes dan gran importancia a la calidad (Caicedo \& Caldas, 2002).

La percepción de lo que significa calidad ha estado presente desde los inicios de la humanidad. La calidad estuvo relacionada con hacer bien las cosas (Maldonado, 2015).

La calidad ha evolucionado desde un enfoque de inspección, pasando por el control, el aseguramiento y llegando a la Gestión Total de la Calidad (TQM - Total Quality Management) (Torres, Ruiz, Solis, \& Martinez, 2012).

En la década de los 90 la Gestión Total de la Calidad (TQM) surgió como respuesta a la cada vez mayores presiones comerciales, Estados Unidos y los países europeos entendieron que la Gestión Total de la Calidad podría ofrecerles una ventaja competitiva con el fin de sobrevivir en el mercado mundial (Merli, 1995) (Jaafreh, 2013).

Esta visión de TQM fue interpretado inicialmente como la necesidad de involucrar a todos los empleados en la mejora de los procesos de las organizaciones; sin embargo en años posteriores se comprendió que TQM significaba que toda la organización debía volcarse a la satisfacción de las necesidades y expectativas de sus clientes, además de sus empleados, accionistas y la sociedad. (Merli, 1995) (Abreu \& Andalia, 1998).

Sin embargo, esta nueva visión necesitaba de una forma concreta y estructurada para poder llevar la filosofía de calidad a una realidad a través de modelos que puedan poner en operación, medir y evaluar la calidad en las organizaciones. (Camisón, Cruz, \& González, 2006). (Chen, Anchecta, Lee, \& Dahlgaard, 2016)

Entre estos modelos encontramos al Iberoamericano de la Excelencia, los premios Deming y Malcom Baldrige, modelo EFQM, Sistema de Gestión de Calidad ISO 9001.

En este trabajo de investigación nos enfocaremos en identificar cuáles son los factores claves del éxito en las organizaciones que tienen implementado un sistema de gestión de calidad según la norma ISO 9001. Para cumplir con este propósito, se revisará la literatura acerca de la Gestión Total de la Calidad (TQM), su relación con la norma ISO 9001 y los factores clave para el éxito de TQM en las organizaciones.

\section{Métodos}

En este artículo fueron utilizados métodos teóricos, el análisis y la síntesis, la revisión de documentos y el histórico lógico. Se revisaron documentados tales como textos, artículos de revistas indexadas y otros cuyos contenidos eran importantes para el desarrollo de este trabajo.

\section{Gestión Total de la Calidad (TQM - Total Quality Management)}

¿Pero qué es Gestión Total de la Calidad o Total Quality Management (TQM)? 
TQM es "esencialmente una forma de organizar y asegurar la participación de toda la empresa, específicamente cada departamento, cada actividad y cada empleado en todos los niveles de la organización" (Chen, Anchecta, Lee, \& Dahlgaard, 2016). La Organización Internacional para la Estandarización (ISO) la definió como "la forma de dirigir una organización, que pretende la participación de todas las personas empleadas para mejorar la calidad de sus productos o servicios, la calidad de sus actividades y la calidad de sus objetivos; con el fin de conseguir la satisfacción de los clientes, la rentabilidad a largo plazo de la organización y los beneficios para las personas empleadas de acuerdo con las exigencias de la sociedad en general." (Socioeconomía). Rahman indica que "TQM es un enfoque de gestión para mejorar el rendimiento de la organización que abarca una variedad de temas técnicos y de comportamiento" (Rahman S, 2005). De igual manera Kaynak define TQM como "una filosofía holística de gestión que se esfuerza por la mejora continua en todas las funciones de una organización" (Kaynak, 2003).

Es una estrategia corporativa que integra los objetivos de la organización con sus propios recursos (Martínez, 2011).

En conclusión, esta filosofía entiende al TQM como un asunto estratégico, una oportunidad de negocio (Gutiérrez, 2010) (Santos \& González, 2008), enfocándose en las necesidades de los consumidores, del mercado y de las partes interesadas (Aldana, y otros, 2011); abandona el concepto de calidad sólo para el proceso productivo para abarcar todas las áreas de la organización bajo un liderazgo claro y visible de la dirección con el compromiso de todo el personal (Cuatrecasas, 2010).

TQM se puede implementar en cualquier tipo de organización y que existe una relación positiva con el desempeño de la empresa (Jaafreh, 2013), lo que significa que TQM permite a las organizaciones alcanzar mayores resultados financieros, operacionales (Kibe \& Wanjau, 2014) y mejorar su posición en el mercado, son estos los motivos que posee una alta aceptación en el campo empresarial (Giménez, Jiménez, \& Martinez, 2014), (Santos \& González, 2008).

Amplia literatura ha identificado los principales beneficios de la gestión total de la calidad: mejora de la calidad de productos y servicios, aumenta la satisfacción de los clientes y empleados, reducción de costos, mejora el desempeño financiero, aumenta la competitividad, incrementa la productividad, aumento de la autonomía de los empleados, incrementa la motivación de los empleados, repercusión en la innovación y la mejora continua (Kibe \& Wanjau, 2014) (Zehir, Ertosun, Zehir, \& Müceldilli, 2012) (Jaafreh, 2013) (Chen, Anchecta, Lee, \& Dahlgaard, 2016) (Martínez, 2011) (Santos \& González, 2008).

Sin embargo, para que TQM pueda ser exitoso y lograr estos beneficios, es necesario una implementación efectiva y decisiones estratégicas acertadas (Kibe \& Wanjau, 2014).

No existe todavía un acuerdo definitivo para determinar una guía general o un camino claro para que su implementación lleve al éxito al TQM (Criado \& Calvo de Mora, 2009) debido a la gran variedad de situaciones que pueden afectar al resultado tales como el contexto de la organización (interno y externo) (Trienekens \& Zuurbier, 2008), (Criado \& Calvo de Mora, 2009), la experiencia en gestión de la calidad de la organización, el tamaño de la empresa o el 
sector de actividad (Criado \& Calvo de Mora, 2009) (Psomas \& Antony, 2015) (Villa, Parroquín, Martínez, \& Torres, 2016). A pesar de lo mencionado, sí ha sido posible definir algunas acciones generales que las organizaciones pueden llevar a cabo (García, 1997). A estas acciones se las ha denominado como Factores críticos o clave para el éxito de la implementación de TQM.

Pero ¿Qué son los factores críticos del éxito? Factores críticos de éxito son prácticas de gestión, acciones o condiciones previas necesarias que garantizan la implementación exitosa de TQM (Jaafreh, 2013).

Según Suárez y Díaz "los factores críticos del éxito se definen como todas aquellas acciones, capacidades, recursos, ventajas, conocimientos y destrezas las cuales constituyen ventaja competitiva" (Suárez \& Díaz, 2013) y en los cuales la organización debería enfocarse (Criado \& Calvo de Mora, 2009). Saraph definió los factores críticos de éxito para TQM como "áreas críticas de planificación y acción gerencial que deben ser practicadas para lograr una gestión eficaz de la calidad en la unidad de negocio" (Gherbal, 2012)

De acuerdo al trabajo de varios investigadores, se pueden resumir los principales factores clave para el éxito de TQM: Liderazgo y compromiso activo de la dirección, desarrollar la gestión adecuada de los recursos humanos (Cultura Organizacional, Formación y Educación), Gestión de procesos, gestión de la calidad de los proveedores, empoderamiento y participación de los empleados, información y análisis, Diseño del producto, Estrategia y Satisfacción del Cliente.(Martínez, 2011) (Gherbal, 2012) (Vouzas, 2016) (Álvarez, Vila, Fraiz, \& Cruz, Análisis de las relaciones de dependencia entre los factores críticos de la calidad y los resultados. Sector de alojamiento turístico en España, 2013) (Zehir, Ertosun, Zehir, \& Müceldilli, 2012) (Giménez, Jiménez, \& Martinez, 2014) (Criado \& Calvo de Mora, 2009) (Rositas, 2009) (Shibani, Saidani, \& Sagoo, 2012) (Jaafreh, 2013) (Wardhani, Utarini, van Dijk, Post, \& Groothoff, 2009) (Álvarez, Vila, Fraiz, \& Cruz, Relación entre herramientas y factores críticos de la calidad, 2014).

\section{Gestión Total de la Calidad (TQM) y la norma ISO 9001.}

La Norma ISO 9001, no es más que un conjunto de requisitos mínimos que, siguiendo la filosofía de la Gestión Total de la Calidad (TQM), debe cumplir una organización para estructurar y poner en marcha un sistema de gestión de calidad eficaz (Marcelino-Ramírez, 2014), es decir que le permita, entre otros fines, la capacidad de proporcionar regularmente productos y servicios que satisfagan los requisitos del cliente y generar oportunidades para aumentar dicha satisfacción (ISO, 2015).

Cantú afirma que "la norma ISO 9001 es una guía para poner en práctica la gestión de calidad total enmarcado en un sistema de gestión." (Cantú, 1997). Muchos autores han considerado que la implementación de la ISO 9001 puede significar el punto de inicio para la aplicación de la Gestión Total de la Calidad (Chen, Anchecta, Lee, \& Dahlgaard, 2016). Revisando la Norma ISO 9001 versión 2015, ésta permite a las organizaciones tres propósitos:

1. Proporcionar productos y servicios que cumplan con los requisitos de los clientes, legales y reglamentarios.

2. Aumentar la satisfacción de los clientes 
3. La mejora continua del sistema de gestión de calidad implementado (ISO, 2015)

4. La norma ISO 9001 se fundamenta en tres estrategias:

5. Enfoque basado en Procesos

6. Ciclo PHVA (Planificar, Hacer, Verificar y Actuar)

7. Pensamiento basado en riesgos. (ISO, 2015)

Para que estas estrategias puedan llevarse a cabo con éxito, la norma ISO 9001 se estructura en 10 capítulos según el Ciclo PHVA:

$\checkmark$ Planificar: Contexto de la Organización (Capítulo 4), Liderazgo (Capítulo 5) y Planificación (Capitulo 6)

$\checkmark$ Hacer: Apoyo (Capítulo 7) y Operación (Capítulo 8)

$\checkmark$ Verificar: Evaluación del Desempeño (Capítulo 9)

$\checkmark$ Actuar: Mejora (Capítulo 10)

Los Capítulos 1, 2 y 3 son sólo aclaratorios y definiciones que no intervienen en la estructuración del Sistema de Gestión de Calidad ISO 9001. (ISO, 2015).

\section{Factores claves para el éxito de los Sistemas de Gestión de Calidad basados en la norma ISO 9001.}

Como se ha visto durante este trabajo, la investigación acerca de los factores críticos o claves para la gestión de la calidad total han sido abundantes, sin embargo no ha ocurrido lo mismo con respecto a la norma ISO 9001 (Psomas \& Antony, 2015).

Villa, Parroquín, Martínez y Torres identifican factores clave sin embargo no son exclusivos para ISO 9001, sino que los generalizan para varios modelos y herramientas de calidad (Villa, Parroquín, Martínez, \& Torres, 2016). En su artículo mencionan el estudio realizado por Kaur y Sharma (2014) quienes indican que los factores críticos que más impactan en los resultados de una organización son la satisfacción del cliente y la satisfacción del empleado. De igual manera aluden a Kutlu y Kadaifci (2012) quienes consideran factores críticos al Compromiso y Liderazgo de la Alta Dirección y la Planificación Estratégica.

Evangelos Psomas \& Jiju Antony analizan en su artículo de manera exclusiva los factores críticos para la eficacia de la norma ISO 9001, quienes identifican a los elementos del entorno interno de la organización (características de la organización, infraestructura, atributos del personal y motivos internos para la certificación ISO 9001) como los que influyen significativamente en el logro de los objetivos de esta norma (Psomas \& Antony, 2015).

Definitivamente es posible encontrar factores claves para el éxito de los sistemas de gestión de calidad basados en ISO 9001 que coincidan con los de la Gestión Total de la Calidad, sin embargo es recomendable que se determinen de manera específica para la norma ISO 9001, debido que éste modelo de gestión tiene características particulares. De esta manera se ha recurrido a la revisión de la norma ISO 9001 versión 2015, tanto en su estructura como a su espíritu, y a diferentes autores que han estudiado esta norma internacional, para establecer los factores claves que permitirán el éxito de su implementación en las organizaciones. A continuación se detallan: 


\section{Visión Sistémica de la organización}

Esta es la característica principal del enfoque a procesos. Las organizaciones constan de sistemas, procesos y actividades (ISO, 2015), a consecuencia de ello la gestión exitosa de una organización depende de la perspectiva de sistemas que posea (Evans \& Lindsay, 2014). El enfoque a procesos tiene que comprenderse y gestionarse desde una visión sistémica, la cual permite a la organización alcanzar los resultados esperados (ISO, 2015) (Criado \& Calvo de Mora, 2009), es decir a través del entendimiento de la interrelación de sus procesos. La norma ISO 9001 promueve la visión sistémica para su correcta implementación y operación (Saumeth, Afanador, Ospino, \& Barraza, 2012).

\section{Orientación a la satisfacción total del cliente}

La calidad la determina el cliente, no la organización (Feigenbaum, 1994) (BerovidesCastellón \& Michelena-Fernández, 2013) por lo que debe enfocarse hacia la satisfacción de las necesidades del cliente, en el presente y en el futuro (Deming, 1986) para exceder sus expectativas, (ISO, 2015) que le va a permitir a las organizaciones, no sólo sobrevivir en el entorno competitivo mundial (Zehir, Ertosun, Zehir, \& Müceldilli, 2012), sino también imponerse.

Esta orientación permanente hacia el cliente estimula la proactividad, es decir al direccionamiento dinámico de los esfuerzos de la organización hacia la identificación constante y sistemática de las necesidades del cliente (Summers, 2006), que permita ofrecerles alternativas de productos y servicios que satisfagan sostenidamente sus expectativas (Santos \& González, 2008) (Aldana, y otros, 2011).

De acuerdo a ISO 9000:2015 - Fundamentos y Vocabulario “Un sistema de gestión de la calidad anima a las organizaciones a analizar los requisitos del cliente, definir los procesos que contribuyen al logro de productos aceptables para el cliente" (ISO, 2015). Por este motivo para que esta filosofía basada en el cliente de los frutos esperados, incluyendo los financieros (Rositas, 2009), las organizaciones deberán priorizar la identificación y planificación de sus procesos clave (Suárez, Roldán, \& Calvo-Mora, 2014), teniendo la capacidad de ser flexibles a los cambios del mercado; reconocer y adaptarse a sus necesidades, que les permitan diseñar productos y servicios que cumplan con los dinámicos requisitos de los clientes y de esta manera satisfacerlos de forma sostenida (Summers, 2006). Precisamente, estas organizaciones son capaces de crear valor porque entienden su cadena de valor, sus procesos clave (Tarí, 2000).

\section{Esfuerzo por aumentar la eficiencia de los procesos}

"La organización debe planificar, implementar y controlar los procesos necesarios para cumplir los requisitos para la provisión de productos y servicios” (ISO, 2015).

La gestión de la calidad permite un enfoque hacia la reducción de costos (BerovidesCastellón \& Michelena-Fernández, 2013), por este motivo no hay que dejar de lado que el esfuerzo para satisfacer totalmente al cliente tiene que ser eficiente, es decir tiene que incluirse la gestión de costos (Summers, 2006). En este caso, las organizaciones que se han preocupado por estructurar procesos que les permita responder rápidamente a las necesidades de sus clientes (especialmente su cadena de suministros), que han promovido una cultura de prevención de errores (Criado \& Calvo de Mora, 2009), que se esfuerzan por mantener sus procesos bajo 
control y disminuir su variación, probablemente obtengan costos más bajos debido a la reducción de desperdicios e ineficiencias (Summers, 2006) (Jaafreh, 2013) (ISO, 2015). En conclusión, el incremento de los beneficios de una organización depende del aumento de las ventas y la reducción de los costos, incluidos los de la no calidad, es decir a través de la eficiencia de la cadena de suministro de la organización.

\section{Planeación estratégica de la calidad}

La adopción de un sistema de gestión de la calidad es una decisión estratégica (ISO, 2015) porque la gestión de la calidad tiene carácter estratégico (Merli, 1995), por este motivo las organizaciones exitosas también tienen en consideración sistemas de planeación para la creación y ejecución de estrategias (Evans \& Lindsay, 2014). Un sistema de gestión de calidad basado en la norma ISO 9001 debe estar continuamente alineado con la dirección estratégica de la organización (ISO, 2015).

El éxito de un sistema de gestión no es asunto del azar, sino que empieza con la planificación y asignación eficiente de recursos (tiempo, dinero, infraestructura, entre otros), del talento humano y el alineamiento de los objetivos estratégicos de la organización (largo plazo) con los de corto plazo (Evans \& Lindsay, 2014) (Jaafreh, 2013).

La planeación estratégica de la calidad permite abandonar la visión de resultados a corto plazo, que usualmente es reactiva, para adoptar una postura de largo plazo (Gallego, 2013) (Jaafreh, 2013) la cual al final genera mejores resultados (Criado \& Calvo de Mora, 2009). En efecto, "planificar es una forma de ver el futuro" (Gallego, 2013).

\section{Liderazgo de la alta dirección}

Deming afirmaba que la alta dirección es responsable de aproximadamente el $80 \%$ de los problemas de calidad en una organización (Villa, Parroquín, Martínez, \& Torres, 2016). El éxito de las organizaciones y por lo tanto de sus sistemas de gestión basados en la norma ISO 9001, depende en gran medida de los estilos de dirección (Baltodano, Arguedas, \& Picón, 2001) (Zúñiga, Campos, \& Cruz, 2001).

Según ISO 9001:2015 Sistemas de gestión de la calidad — Requisitos, los líderes establecen la unidad de propósito y la orientación de la organización (ISO, 2015). La alta dirección debe demostrar liderazgo y compromiso hacia el sistema de gestión de calidad y hacia el enfoque al cliente; debe establecer, implementar y mantener una política de la calidad y debe asegurarse de que las responsabilidades y autoridades para los roles pertinentes se asignen, se comuniquen y se entiendan en toda la organización. (ISO, 2015).

El liderazgo y el compromiso de la alta dirección son de gran importancia para mantener la orientación hacia la satisfacción del cliente (Campos, Mendes, Silva, \& Valle, 2014).

Para que los sistemas de gestión de calidad basados en la ISO 9001 influyan positivamente en los sistemas de gestión de calidad en cuanto al desempeño organizacional, en la satisfacción del cliente y en los resultados financieros (Rositas, 2009) (Villa, Parroquín, Martínez, \& Torres, 2016) (Kibe \& Wanjau, 2014) (Álvarez, Vila, Fraiz, \& Cruz, Análisis de las relaciones de dependencia entre los factores críticos de la calidad y los resultados. Sector de 
alojamiento turístico en España, 2013) Se requiere que la alta dirección demuestre un liderazgo directo, activo y comprometido (Merli, 1995) (ISO, 2015).

No es suficiente sólo decir que la dirección apoya, sino que tiene que notarse, tiene que sentirse (Deming, 1986). Entre las principales características de los líderes actuales tenemos su capacidad de conducir a la organización hacia una cultura centrada en la calidad (Tarí, 2000) (Summers, 2006) y a través de una visión compartida (Campos, Mendes, Silva, \& Valle, 2014), garantizar un buen clima laboral, que permita a los trabajadores aportar al sistema de gestión de calidad, en la innovación y su mejora continua (Aldana, y otros, 2011) (Zehir, Ertosun, Zehir, \& Müceldilli, 2012).

Otra característica de los líderes es alinear las necesidades, requerimientos y expectativas del cliente con la planeación estratégica de la organización e impulsar a los empleados a que las iniciativas que se desprendan se entrelacen con esta planeación (Summers, 2006). El liderazgo de la alta dirección asume y promueve la planeación estratégica de calidad para alcanzar la satisfacción al cliente y los resultados de la organización a largo plazo (Jaafreh, 2013) (Kibe \& Wanjau, 2014) (Caicedo \& Caldas, 2002).

De igual manera, los líderes eficaces hablan con sus actos, es decir no sólo dice a los demás lo que tienen que hacer, sino que él es el primero que hace y con estas acciones deja en claro a su personal lo que espera de ellos (Summers, 2006). Si no existe coherencia entre lo que se dice y lo que se hace, el líder irremediablemente perderá credibilidad y confianza, de la misma manera que el sistema de gestión de calidad (Caicedo \& Caldas, 2002). Como dice aquella popular frase: "Las palabras convencen pero el ejemplo arrastra".

\section{Compromiso del personal}

El personal es el motor de todo sistema de gestión de calidad, es la esencia de la organización, sus habilidades permiten que se ocupen de que los requisitos de los productos y servicios cumplan las especificaciones y satisfagan a los clientes. (ISO, 2015). Por este motivo la alta dirección ha de generar todas las facilidades para que exista una comunicación efectiva y una organización del trabajo que permitan crear el ambiente adecuado para la participación de todos los empleados en la gestión de la calidad y garanticen su eficacia (Aldana, y otros, 2011) (Grijalvo \& Prida, 2011), (ISO, 2015). Las organizaciones que no se esmeren de manera suficiente en motivar a sus empleados para participar en la solución de los problemas de calidad, no lograrán que se comprometan con su sistema de gestión ISO 9001 (Álvarez, Vila, Fraiz, \& Cruz, Análisis de las relaciones de dependencia entre los factores críticos de la calidad y los resultados. Sector de alojamiento turístico en España, 2013).

El gurú de la calidad Philip Crosby afirmaba que "La colaboración y el trabajo en equipo son esenciales en el desarrollo de la gestión de la calidad" (Aldana, y otros, 2011). De igual manera Armand V. Feigenbaum reiteraba que "La calidad no es un problema aislado, abarca a toda la organización" (Aldana, y otros, 2011), por lo tanto el trabajo en equipo influye positivamente en el desempeño organizacional (Campos, Mendes, Silva, \& Valle, 2014).

\section{El aumento de la competencia del personal}


Según la Norma ISO 9000: 2015 - Vocabulario, competencia "es la aptitud demostrada para aplicar los conocimientos y habilidades con el fin de lograr los resultados previstos" (ISO, 2015). El nivel de competencia se basa en la educación, formación o experiencia apropiadas (ISO, 2015).

El aumento de la competencia del talento humano se considera uno de los factores más importantes para la eficacia para un SGC (Psomas \& Antony, 2015), (Kibe \& Wanjau, 2014), (Gherbal, 2012). La calidad empieza y termina con la formación (Benitez \& Mercedes, 2007) es decir que la formación debe estar presente en todo momento, y por lo tanto dirigida a todas las dimensiones de la persona y a todos los niveles de la organización, es decir debe ser integral.

Cuando la organización se esfuerza por aumentar la competencia de su personal, se generan productos y servicios de mayor calidad reduciendo los desperdicios y por lo tanto los costos (Vouzas, 2016), fortalece su capacidad creativa e innovadora (Kibe \& Wanjau, 2014) e impulsa la mejora continua en la organización (Álvarez, Vila, Fraiz, \& Cruz, Análisis de las relaciones de dependencia entre los factores críticos de la calidad y los resultados. Sector de alojamiento turístico en España, 2013).

Para que la competencia contribuya al éxito de los Sistemas de Gestión, los planes a desarrollar no sólo deben considerar la adquisición de conocimientos, sino además dirigirse hacia el fortalecimiento del hábito (destrezas), la conciencia y la motivación, que permitan la generación de una cultura de calidad. Para lograrlo es fundamental que dichos planes sean estructurados y sistemáticos, con enfoque al mediano y largo plazo, con objetivos específicos, actividades con sus responsabilidades definidas, con una metodología que abarque las diferentes culturas, educación y jerarquía organizacional de los empleados, con un seguimiento estricto a los resultados que se vayan obteniendo (Merli, 1995) (Caicedo \& Caldas, 2002). De igual manera es necesario que los empleados tengan una actitud de apertura hacia el aprendizaje. Las organizaciones con mayor capacidad de aprender, se adaptan mejor y más rápido a los cambios internos como externos y pueden por lo tanto, obtener mejores resultados y en el menor tiempo (Criado \& Calvo de Mora, 2009).

\section{Empoderamiento de la calidad}

El empoderamiento se define como "la capacidad del individuo para influir y controlar su propio ambiente de trabajo y esforzarse por lograr más autodeterminación" Empoderar significa transferir autoridades y responsabilidades de un nivel jerárquico superior a uno inferior (Campos, Mendes, Silva, \& Valle, 2014) (Grijalvo \& Prida, 2011), lo que permite que puedan detectar y a su vez resolver los problemas de calidad que se presenten (Campos, Mendes, Silva, \& Valle, 2014), tomando decisiones oportunas (Jaafreh, 2013) y logrando, de esta manera, mejorar constantemente los resultados de sus actividades y por lo tanto del sistema de gestión de calidad. (Grijalvo \& Prida, 2011).

Hay que abandonar la idea que la calidad depende de la inspección, sólo se puede entregar productos y servicios de calidad a los clientes de manera sostenida si se mejoran los procesos (Deming, 1986). 
Dejar la calidad en manos de la inspección es reconocer que los encargados de generar los productos y/o servicios no son capaces de cumplir los requisitos que el cliente demande (Deming, 1986). Según Feigenbaum cada miembro de la organización debe controlar su propio proceso y ser totalmente responsable de la calidad de los productos y/o servicios que realizan (Berovides-Castellón \& Michelena-Fernández, 2013). La formación en calidad y la progresiva culturización deben conducir al empoderamiento de la calidad de las actividades de los empleados (Caicedo \& Caldas, 2002). La inspección es costosa, por un lado por el número de personas que deben sumarse a la organización y por el otro lado, por el reproceso, la eliminación de los productos no conformes (Deming, 1986) y el costo de oportunidad por la inadecuada utilización de los recursos. "El grado de implementación del SGC será mayor en proporción al grado de empleados empoderados" (Wardhani, Utarini, van Dijk, Post, \& Groothoff, 2009).

\section{Conclusión}

Durante este trabajo de investigación, se lograron definir factores claves que las organizaciones deben tomar en cuenta para que sus sistemas de gestión de calidad basados en la norma ISO 9001 tengan el éxito y permitan obtener los resultados que la organización requiere: Visión sistémica de la organización, orientación a la satisfacción total del cliente, esfuerzo por aumentar la eficiencia de los procesos, planeación estratégica de la calidad, liderazgo de la alta dirección, compromiso del personal, el aumento de la competencia del personal y empoderamiento de la calidad.

Estos factores fueron definidos después de una revisión de las normas ISO 900, ISO 9000 y diferentes artículos. Existe similitud en la mayoría de los factores identificados con los de la Gestión de la Calidad Total, sin embargo la diferencia radica en el propósito que persigue la ISO 9001 y en su estructura. De todas formas hay que reconocer que probablemente existan otros factores que este trabajo no ha considerado.

Estos factores claves no se los debe tratar de manera independiente, sino reconociendo la sinergia existente entre ellos; por este motivo se convierte en un requisito fundamental para que la aplicación de estos factores en la organización sea exitosa. Por ejemplo, es cada vez más evidente que no se puede excluir al factor humano de la gestión de la calidad. El compromiso, el empoderamiento y el aumento de competencia de los empleados son piezas clave para el desarrollo de una cultura de la innovación. La innovación no es más que el resultado de la capacidad de la organización por identificar e interpretar constantemente las necesidades del mercado, que la lleva a generar iniciativas de nuevos productos y servicios que cumplan con sus expectativas, es decir de la capacidad de la organización de mejorar continuamente.

Para que la innovación sea sustentable, se requieren de procesos con enfoque sistémico, eficientes y robustos, que permitan sostener las operaciones de la organización, optimizando el uso de los recursos, reduciendo los costos y las actividades que no agreguen valor para el cliente. La alta dirección debe tener un liderazgo activo, comprometido, integrador y estratégico que impacte decisivamente en la calidad de los productos y servicios en la satisfacción del cliente, en los resultados operacionales y financieros.

El impacto del liderazgo de la alta dirección se transmite por medio de la participación y competencia del personal de la organización. Este artículo deja la puerta abierta para que se 
estudie la correlación entre estos factores clave del éxito y el grado de impacto en los resultados de la organización. De igual manera se podría investigar la eficacia de estos factores clave de acuerdo a las características de las organizaciones (tamaño, actividad económica, ventas, etc).

\section{Bibliografía}

Abreu, M. d., \& Andalia, R. C. (1998). Gerencia Total de la Calidad en las organizaciones. ACIMED.

Aldana, L., Álvarez, M., Bernal, C., Díaz, M., González, C., Galindo, Ó., \& Villegas, A. (2011). Administración por Calidad. Bogotá: Editorial Alfaomega .

Álvarez, J., Vila, M., Fraiz, J. A., \& Cruz, M. d. (2013). Análisis de las relaciones de dependencia entre los factores críticos de la calidad y los resultados. Sector de alojamiento turístico en España. Investigaciones Europeas de Dirección y Economía de la Empresa, 74-89.

Álvarez, J., Vila, M., Fraiz, J. A., \& Cruz, M. d. (2014). Relación entre herramientas y factores críticos de la calidad. Revista Europea de Dirección y Economía de la Empresa, 82-97.

Baltodano, V., Arguedas, C., \& Picón, J. C. (2001). Enfoques y estilos gerenciales modernos adaptados a ambientes internacionales. Revista Geográfica de América Central, 75-91.

Benitez, F., \& Mercedes, A. (2007). Guía administrativa para implementar el sistema de gestión de calidad en las PYMES en Boyacá. Semestre Económico, 101-112.

Berovides-Castellón, M., \& Michelena-Fernández, E. (2013). La gestión de la calidad en una empresa de pastas alimenticias. Ingeniería Industrial/, 252-266.

Caicedo, S., \& Caldas, M. (2002). La importancia de la cultura organizacional en la implementación y mantenimiento de sistemas de gestión de calidad basados en la norma ISO 9000. Innovar, 135-148.

Camisón, C., Cruz, S., \& González, T. (2006). Gestión de la Calidad: Conceptos, enfoques, modelos y sistemas. Madrid: Pearson Educación.

Campos, A. C., Mendes, J. d., Silva, J. A., \& Valle, P. O. (2014). Critical success factors for a total quality culture: A structural model. Tourism \& Management Studies, 7-15.

Cantú, H. (1997). Desarrollo de una Cultura de Calidad. México: McGraw-Hill/Interamericana Editores S.A.

Chen, C.-k., Anchecta, K., Lee, Y.-D., \& Dahlgaard, J. J. (2016). A Stepwise ISO-based TQM Implementation Approach. Management and Production Engineering Review, 65-75.

Criado, F., \& Calvo de Mora, A. (2009). Factores clave y rendimiento en empresas españolas con sistemas de gestión de la calidad. Revista Europea de Dirección y Economía de la Empresa, 107-126.

Cuatrecasas, L. (2010). Gestión Integral de la Calidad: Implantación, Control y Certificación. Bresca.

Deming, W. E. (1986). Ot of the Crisis. Cambridge: MIT-CAES.

Evans, J., \& Lindsay, W. (2014). Administración y Control de la Calidad (9na ed.). Monterrey: Cengage Learning. 
Feigenbaum, A. (1994). Control Total de la Calidad. México: Editorial Continental.

Gallego, G. (29 de Diciembre de 2013). Free E-Books. (F. E-Books, Ed.) Recuperado el abril de 2017, de http://espanol.free-ebooks.net/ebook/La-Calidad-mas-Alla-de-la-Certificacion

García, J. (1997). Calidad Total. Málaga: Universidad de Málaga.

Gherbal, N. (2012). Critical Success Factors of Implementing Total Quality Management in Libyan Organisations. Proceedings of the 2012 International Conference on Industrial Engineering and Operations Management, 80-89.

Giménez, J. A., Jiménez, D., \& Martinez, M. (2014). La gestión de calidad: importancia de la cultura organizativa para el desarrollo de variables intangibles. Revista Europea de Dirección y Economía de la Empresa, 115-126.

Grijalvo, M., \& Prida, B. (2011). Desarrollo de un modelo conceptual para la implantación de sistemas de calidad en sistemas sociotécnicos. XV Congreso de Ingeniería de Organización, 143-153.

Gutiérrez, H. (2010). Calidad total y productividad. México: Mc Graw-Hill - Interamericana Editores, S.A.

ISO. (2015). ISO 9000:2015 Sistemas de gestión de la calidad —Fundamentos y vocabulario. Ginebra, Suiza: ISO.

ISO. (2015). ISO 9001:2015 Sistema de Gestión de la Calidad - Requisitos. ISO. Ginebra, Suiza.

Jaafreh, A. B. (2013). The Effect of Quality Management Practices on Organizational. Sciedu Press, 93109.

Kaynak, H. (2003). The relationship between total quality management practices and their effects on firm performance. Journal of Operations Management 21, 405-435.

Kibe, E. N., \& Wanjau, D. K. (2014). The Effect of Quality Management Systems on the Performance of Food Processing Firms in Kenya. IOSR Journal of Business and Management, 61-72.

Maldonado, J. A. (2015). Fundamentos de Calidad Total.

Marcelino-Ramírez. (2014). Administración de la Calidad. Nuevas Perspectivas. México: Grupo Editorial Patria S.A.

Martínez, R. (2011). Modelos para la Implementación de la Gestión de la Calidad Total en las PYMES Latinoamericanas. Gestión y Gerencia, 68-86.

Merli, G. (1995). La Calidad Total como Herramienta de Negocio. México: Diaz de Santos.

Psomas, E., \& Antony, J. (2015). The effectiveness of the ISO 9001 quality management system and its influential critical factors in Greek manufacturing companies. International Journal of Production Research, 2089-2099.

Rahman S, B. P. (2005). Soft TQM hard TQM and organizational performance relationships: an empirical investigation. Omega 33, 73-83. 
Rositas, J. (2009). Factores críticos de éxito en la gestión de calidad total en la industria manufacturera mexicana. Ciencia UANL, 181-193.

Santos, M. L., \& González, L. I. (2008). Efectos de la Gestión de Calidad Total. Cuadernos de Economía y Dirección de la Empresa., págs. 033-066.

Saumeth, K. M., Afanador, T. S., Ospino, L. S., \& Barraza, F. M. (2012). Calidad y su evolución: una revisión. Dimens. empres., 100-107.

Shibani, A., Saidani, M., \& Sagoo, A. (2012). Critical Success Factors of Implementing Total Quality Management in Libyan Organisations. International Conference on Industrial Engineering and Operations Management, 80-89.

Socioeconomía, B. d. (s.f.). Cuaderno para emprendedores y empresarios: gestión de la calidad total. Sevilla : Sevilla Siglo XXI, S.A.

Suárez, E., Roldán, J., \& Calvo-Mora, A. (2014). A structural analysis of the EFQM model: an assessment of the mediating role of process management. Journal of Business Economics and Management, $862-885$.

Suárez, W., \& Díaz, J. (2013). Factores críticos del éxito en la investigación universitaria. Conference: I Congreso Iberoamericano sobre Gobernanza Universitaria, 108-118.

Summers, D. (2006). Administración de la calidad. México: Pearson Educación.

Tarí, J. (2000). Calidad total: fuente de ventaja competitiva. Murcia: Publicaciones Universidad de Alicante.

Torres, K. T., Ruiz, T., Solis, L., \& Martinez, F. (2012). Calidad y su evolución: una revisión. Dimensión Empresarial, 100-107.

Trienekens, J., \& Zuurbier, P. (2008). Quality and Safety Standards in the Food Industry, Developments and Challenges. International Journal of Production Economics, 107-122.

Villa, A. J., Parroquín, P. C., Martínez, E. A., \& Torres, V. (2016). Factores críticos del éxito de los sistemas de gestión de calidad:. Culcyt // Calidad en la Salud, 78-87.

Vouzas, F. (2016). ISO 9001 and Business Excellence Models - How "Human" they are? the case in Greece. (C) 20-ICIT, 1-9.

Wardhani, V., Utarini, A., van Dijk, J. P., Post, D., \& Groothoff, J. W. (2009). Determinants of quality management systems implementation. Health Policy, 239-251.

Zehir, C., Ertosun, Ö. G., Zehir, S., \& Müceldilli, B. (2012). Total Quality Management Practices Effects on Quality Performance and Innovative Performance. Procedia - Social and Behavioral Sciences, $273-280$.

Zúñiga, V. H., Campos, C. A., \& Cruz, J. C. (2001). Enfoque y estilos Gerenciales Modernos adaptados a ambientes internacionales. Revista Geográfica de América Central, 75-91. 